\title{
THE LEBESGUE DECOMPOSITION OF MEASURES ON ORTHOMODULAR POSETS
}

\author{
By G. T. RÜTTIMANN and CHR. SCHINDLER*
}

[Received 14th October 1985]

\section{Introduction}

THE study of measures on orthomodular posets has its origin in the quantum logic approach to quantum mechanics $[2,5,6,12,15]$ and as a mathematical branch it became known as non-commutative measure theory (e.g. see [17]).

The purpose of this paper is to investigate the Lebesgue decomposition of positive measures into positive measures in this non-commutative setting. The geometrical aspect of this notion is emphasized.

The main theorems in $\$ 3(3.4 \& 3.5)$ present a condition for subcones of the cone of positive measures on an orthocomplete orthomodular poset $L$ under which the requirement of a positive Lebesgue decomposition is equivalent to the poset $L$ to be a Boolean lattice. These conditions are met by the cone obtained by restricting normal positive linear functionals on a $J B W$-algebra to the complete orthomodular lattice of idempotents in this algebra. As an application of the aforementioned result we obtain a measure-theoretic characterization of associative $J B W$-algebras amongst all $J B W$-algebras. This is the main result of $\$ 5$. Paragraph 4 is concerned with certain permanence properties of the positive Lebesgue decomposition, i.e. the behaviour of this decomposition for selected sets of probability measures under the formation of direct products and direct sums. In particular it is shown that the collection of all probability measures of a finite constructible orthomodular poset has the positive Lebesgue decomposition property.

\section{Prerequisites}

Let us begin with the definitions and basic facts which pertain to this paper.

Let $\left(L, \leqslant,,^{\prime}\right)$ be an orthocomplemented poset, ${ }^{\#} L>1$, with 0 as the least and 1 as the greater element. A pair $(p, q)$ of elements of $L$ is said to be orthogonal, denoted by $p \perp q$, provided $p \leqslant q^{\prime}$. A subset $D$ of $L$ is said to be orthogonal if each pair $(p, q)$ of $D$ with $p \neq q$ is orthogonal. Clearly, a subset $C$ of $L$ has at most one supremum, resp. infimum. We denote the supremum of $C$, resp. infimum of $C$, if such exists, by

\footnotetext{
- Research supported by Schweizerischer Nationalfonds/Fonds nationalsuisse under grant numbers $2.807-0.83,2.200-0.84$.
}

Qwart. J. Math. Oxford (2), 37 (1986), 321-345

(C) 1996 Oxford University Press 
sup $C=\bigvee C$, resp. $\inf C=\wedge C$. The following notations are also used: $p \vee q=\sup \{p, q\}, \quad p \wedge q=\inf \{p, q\}, \bigvee_{i \in I} p_{i}=\sup \left\{p_{i}: i \in I\right\}, \quad \bigwedge_{i \in I} p_{i}=$ $\inf \left\{p_{i}: i \in I\right\}$.

By an orthomodular poset $[4,13,17]$ we mean an orthocomplemented poset $(L, \leqslant, ')$ satisfying the following conditions:

(i) if $p \perp q$ then $p \vee q$ exists,

(ii) if $p \perp q$ and $p \vee q=1$ then $p=q^{\prime}$.

In an orthocomplemented poset and in presence of (i) condition (ii) is equivalent with

(ii') if $p \leqslant q$ then $q=p \vee\left(q \wedge p^{\prime}\right)$, noticing that the right-hand side exists.

An orthomodular poset is said to be orthocomplete, resp. $\sigma$ orthocomplete, if the supremum of every orthogonal subset, resp. countable orthogonal subset, exists. If an orthomodular poset is indeed a lattice then it is referred to as an orthomodular latice. Notice that an orthocomplete, resp. $\sigma$-orthocomplete, orthomodular lattice is complete, resp. $\sigma$-complete $[\mathbf{9}, 21]$.

Let $(L, \leqslant, ')$ be an orthomodular poset. A pair $(p, q)$ of elements of $L$ is said to be compatible, denoted by $p C q$, provided there exist elements $u, v$ and $w$ with $u \perp v \perp w \perp u$ such that

$$
p=u \vee v
$$

and

$$
q=u \vee w
$$

Notice that for elements $p$ and $q$ of $L$

$$
p C q \Leftrightarrow q C p \Leftrightarrow p C q^{\prime}
$$

holds true. Also, $p C q$ if and only if $p \wedge q$ and $p \wedge q^{\prime}$ both exist and

$$
p=(p \wedge q) \vee\left(p \wedge q^{\prime}\right) .
$$

If all pairs of elements of $L$ are compatible then the orthomodular poset $\left(L, \leqslant,,^{\prime}\right)$ is said to be Boolean. One verifies that in this case $\left(L, \leqslant,,^{\prime}\right)$ is indeed a Boolean lattice.

Let $(L, \leqslant, ')$ be an orthomodular poset. An element $\mu$ of the product vector space $\mathbb{R}^{L}$ is said to be a measure on $L$ if $p \perp q$ implies that

$$
\mu(p \vee q)=\mu(p)+\mu(q) .
$$

A measure $\mu$ is said to be positive if $\mu(p) \geqslant 0$ for all $p \in L$. The collection of positive measures on $L$, denoted by $K(L)$, forms a cone in $\mathbb{R}^{L}$. By orthomodularity, a positive measure on $L$ is an isotonic map on the poset 
$(L, \leqslant)$. A measure $\mu$ is said to be normalized if

$$
\mu(1)=1 \text {. }
$$

Finally, a positive and normalized measure is called a probability measure on $L$. The collection of probability measures on $L$, denoted by $\Omega(L)$, is a convex and $\tau$-compact subset of $\mathbb{R}^{L}$ where $\tau$ is the product topology on $\mathbb{R}^{L}$. Moreover, $K(L)$ coincides with the positive hull of $\Omega(L)$.

A measure $\mu$ is called completely additive, resp. $\sigma$-additive, if for every orthogonal, resp. countable orthogonal, subset $D$ of $L$ for which $\bigvee D$ exists

$$
\mu(\bigvee D)=\lim (\mu(\vee C))_{C \in D^{\prime}}
$$

holds true, where $\left(D^{f}, \subseteq\right)$ denotes the collection of finite subsets of $D$ directed by set-inclusion. With $\Omega_{c}(L)$, resp. $\Omega_{\sigma}(L)$, we denote the set of completely additive, resp. $\sigma$-additive, probability measures on $L$. Notice that both $\Omega_{c}(L)$ and $\Omega_{\sigma}(L)$ are faces of $\Omega(L)$. For details of these and other properties of measures on orthomodular posets the reader is referred to $[3,19]$.

A positive measure $\mu$ on an orthomodular poset $\left(L, \leqslant,,^{\prime}\right)$ is said to be a Jauch-Piron measure [18] provided that every finite subset of the kernel of $\mu$, denoted $\operatorname{ker} \mu$, has an upper bound in $\operatorname{ker} \mu$. The collection of normalized Jauch-Piron measures is denoted by $\Omega_{J P}(L)$. If $(L, \leqslant, ')$ is an orthomodular lattice and $\mu \in \Omega(L)$, then $\mu \in \Omega_{J P}(L)$ if and only if

$$
\mu(p)=\mu(q)=1
$$

implies that

$$
\mu(p \wedge q)=1 .
$$

Accordingly, we define a positive measure $\mu$ to be a c-Jauch-Piron, resp. $\sigma$-Jauch-Piron, measure if every subset, resp. every countable subset, of $\operatorname{ker} \mu$ has an upper bound in $\operatorname{ker} \mu$. Then $\Omega_{c . J P}(L)$, resp. $\Omega_{\sigma . J P}(L)$, denotes the collection of all normalized $c$-Jauch-Piron, resp. $\sigma$-JauchPiron, measures on $L$.

Relations between the various notions of a Jauch-Piron measure and higher-order additivity of probability measures are given below. Notice that part (ii) is an extension of [10, Theorem 9].

TheOREM 2.1. Let $\left(L, \leqslant,,^{\prime}\right)$ be an orthomodular poset.

(i) If $\left(L, \leqslant,,^{\prime}\right)$ is $\sigma$-orthocomplete then

$$
\boldsymbol{\Omega}_{\sigma}(L) \cap \boldsymbol{\Omega}_{J P}(L) \subseteq \boldsymbol{\Omega}_{\sigma \cdot J P}(L) ;
$$

(ii) if $\left(L, \leqslant,,^{\prime}\right)$ is orthocomplete then

$$
\boldsymbol{\Omega}_{c}(L) \cap \boldsymbol{\Omega}_{J P}(L) \subseteq \boldsymbol{\Omega}_{c \cdot J P}(L) ;
$$


(iii) if $\left(L, \leqslant,,^{\prime}\right)$ is orthocomplete or is a $\sigma$-complete orthomodular lattice (equivalently: $\sigma$-orthocomplete orthomodular lattice) then

$$
\Omega_{o}(L) \cap \Omega_{c-\mathcal{P}}(L) \subseteq \Omega_{c}(L)
$$

Proof. (i) Let $\mu$ be an element of $\Omega_{\sigma}(L) \cap \Omega_{J P}(L)$ and let $E$ be a countable non-empty subset of ker $\mu$. Let $\left(p_{i}\right)_{i \in N}$ be an enumeration of $E$. We define the isotone sequence $\left(q_{i}\right)_{i \in \mathrm{N}}$ as follows:

$q_{1}:=p_{1}$ and $q_{i} \in \operatorname{ker} \mu$ with $q_{i} \geqslant q_{1}, q_{2}, \ldots, q_{i-1}, p_{i}$

$$
\text { for } i=2,3, \ldots \text {. }
$$

Moreover, we define

$$
r_{1}:=q_{1}, \quad r_{i}:=q_{i} \wedge q_{i-1}^{\prime} \text { for } i=2,3, \ldots
$$

Now $r_{i} \perp r_{j}$ provided that $i \neq j$ and

$$
q_{j}=\bigvee_{i=1}^{j} r_{i}
$$

for $j=1,2, \ldots$.

Therefore $\bigvee_{i=1}^{\infty} r_{i}$ is an upper bound for $E$. Also, since $\mu$ is a $\sigma$-additive probability measure, we conclude that

$$
\mu\left(\bigvee_{i=1}^{\infty} r_{i}\right)=\lim _{j \rightarrow \infty} \mu\left(\bigvee_{i=1}^{j} r_{i}\right)=0
$$

(ii) Let $\mu$ be an element of $\Omega_{c}(L) \cap \Omega_{j P}(L)$. By Zorn's lemma, there exists a maximal orthogonal set $D$ in $\operatorname{ker} \mu$; set $p:=\bigvee D$. Then

$$
\mu(p)=\lim (\mu(\vee C))_{C \in D^{\prime}}=0,
$$

hence $p$ is an element of ker $\mu$.

Now let $r$ be an element of $\operatorname{ker} \mu$. Then there exists an element $s$ in ker $\mu$ which majorizes both elements $p$ and $r$. By orthomodularity, we get

$$
0=\mu(s)=\mu(p)+\mu\left(p^{\prime} \wedge s\right)=\mu\left(p^{\prime} \wedge s\right) .
$$

But $p^{\prime} \wedge s \perp D$ and by maximality of $D$ in $\operatorname{ker} \mu$ we conclude that $p^{\prime} \wedge s$ is the least element. Therefore $p$ coincides with $s$ which is an upper bound for $r$.

(iii) Let $\mu$ be an element of $\Omega_{o}(L) \cap \Omega_{c-J P}(L)$ and let $E$ be a maximal orthogonal set in $L$. Then the set

$$
F:=\{p \in E: \mu(p) \neq 0\}
$$

is countable. 
Assume $\left(L, \leqslant,,^{\prime}\right)$ to be an orthocomplete orthomodular poset and define

$$
q:=\bigvee(E-F)
$$

Again by orthomodularity, we then conclude that $q^{\prime}$ is the supremum of F.

Since $\mu$ is an element of $\Omega_{c \downarrow J P}(L)$ the set ker $\mu$ contains an upper bound for $E-F$. Therefore $q$ is an element of $\operatorname{ker} \mu$ and

$$
\begin{aligned}
1=\mu(q)+\mu\left(q^{\prime}\right) & =\lim (\mu(\bigvee C))_{C \in F^{\prime}} \leqslant \sup _{D \in E^{\prime}} \mu(\bigvee D) \\
& =\lim (\mu(\bigvee D))_{D \in E^{\prime}} \leqslant 1 .
\end{aligned}
$$

Hence $\mu$ is a completely additive measure.

Suppose now that $\left(L, \leqslant,,^{\prime}\right)$ is a $\sigma$-complete orthomodular lattice and set

$$
r:=\bigvee F \text {. }
$$

Clearly, $r^{\prime}$ is an upper bound of the set $E-F$ and for any upper bound $s$ of $E-F$ we then have

$$
E-F \perp r^{\prime} \wedge\left(r \vee s^{\prime}\right) \perp r
$$

Therefore $E \perp r^{\prime} \wedge\left(r \vee s^{\prime}\right)$ and by maximality of $E$ we get

$$
r^{\prime} \wedge\left(r \vee s^{\prime}\right)=0
$$

or equivalently,

$$
r^{\prime} \leqslant s
$$

To this end we have shown that $r^{\prime}$ is the supremum of the set $E-F$. But $\mu$ is an element of $\Omega_{c-J P}(L)$, hence $r^{\prime}$ is an element of ker $\mu$. The conclusion is now reached by precisely the same arguments as above.

\section{The Positive Lebesgue Decomposition}

Let $\left(L, \leqslant,,^{\prime}\right)$ be an orthomodular poset and, again, let $K(L)$ denote the cone of positive measures on $L$. As in classical measure theory we define the pair $(\lambda, \mu)$ of elements of $K(L)$ to be singular, denoted by $\lambda \perp \mu$, if there exists an element $p$ in $L$ such that

$$
\lambda(p)=0=\mu\left(p^{\prime}\right) .
$$

An element $\lambda$ of $K(L)$ is sair to be absolutely continuous with respect to an element $\mu$ of $K(L)$, denoted by $\lambda \ll \mu$, provided that

$$
\mu(p)=0 \quad \text { implies that } \lambda(p)=0 .
$$


Let $\Delta$ be a convex subset of the convex set $\Omega(L)$ of probability measures on $L$. One verifies that the positive hull of $\Delta$, denoted by $K(\Delta)$, is a subcone of $K(L)$. If $\Delta$ is non-empty then $K(\Delta)=\mathbb{R}_{+} \cdot \Delta$. We say that $\Delta$ has the positive Lebesgue decomposition property (PLD) provided that for each pair $(\lambda, \mu)$ of elements of $K(\Delta)$ there exists a pair $(v, \xi)$ of elements of $K(\Delta)$ such that

$$
v \perp \lambda, \quad \xi \ll \lambda
$$

and

$$
\mu=v+\xi
$$

For each element $p$ in $L$ we denote with $a_{\Delta}(p)$ the subset $\{\mu \in$ $\Delta: \mu(p)=1\}$; notice that $a_{\Delta}(p)$ is a face of $\Delta$. We are now in a position to give a "geometrical form" of the positive Lebesgue decomposition property.

LEMMA 3.1. Let $\left(L, \leqslant,,^{\prime}\right)$ be an orthomodular poset and $\Omega(L)$ the convex set of probability measures on $L$. Let $\Delta$ be a convex subset of $\Omega(L)$.

Then $\Delta$ has the positive Lebesgue decomposition property if and only if for each element $\lambda$ of $\Delta$ the set

$$
\bigcup_{q \in \text { ter } \lambda} \operatorname{conv}\left(\bigcap_{p \in \operatorname{ker} \lambda} a_{\Delta}\left(p^{\prime}\right) \cup a_{\Delta}(q)\right)
$$

coincides with $\Delta$.

Proof. Suppose that $\Delta$ has the PLD. Let $\lambda, \mu$ be elements of $\Delta$ and let $(v, \xi)$ be a pair of elements in $K(\Delta)$ establishing the desired decomposition with respect to the pair $(\lambda, \mu)$.

We first assume that $v$ and $\xi$ are different from 0 . Then

$$
v(1), \xi(1)>0 \text { and } v(1)+\xi(1)=1
$$

and, moreover, the elements $v / v(1), \xi / \xi(1)$ belong to $\Delta$. We then have the following representation

$$
\mu=v(1)(v / v(1))+\xi(1)(\xi / \xi(1)) .
$$

Clearly, for an element $p$ in $\operatorname{ker} \lambda$ we get

$$
(\xi / \xi(1))\left(p^{\prime}\right)=1 \text {. }
$$

Thus $\xi / \xi(1)$ is a member of the set $\bigcap_{p \in \text { ter } \lambda} a_{\Delta}\left(p^{\prime}\right)$. Also, there exists an element $q$ in $L$ such that

$$
v(q)=0=\lambda\left(q^{\prime}\right)
$$


Thus $q^{\prime}$ is a member of ker $\lambda$ and $v / v(1)$ belongs to $a_{\Delta}\left(q^{\prime}\right)$. The cases where $v$ or $\xi$ are equal to 0 are readily dealt with. This proves necessity of the given condition.

The converse is straightforward.

As an immediate consequence, we note that the positive Lebesgue decomposition property is facially descendingly hereditary.

Corollary 3.2. Let $\left(L, \leqslant,^{\prime}\right)$ be an orthomodular poset and let $\Delta$ be a convex subset of $\Omega(L)$. If $\Delta$ has the positive Lebesgue decomposition property, then so has each of its faces.

Proof. Let $\Delta^{\prime}$ be a face of $\Delta$. Then

$$
\Delta^{\prime}=\Delta^{\prime} \cap \Delta=\bigcup_{q \in \text { Ker } \mu} \operatorname{conv}\left(\bigcap_{p \in \text { ker } \mu}\left(\Delta^{\prime} \cap a_{\Delta}\left(p^{\prime}\right)\right) \cup\left(\Delta^{\prime} \cap a_{\Delta}(q)\right)\right),
$$

equality holding since

$$
\bigcap_{p \in \operatorname{ker} \mu} a_{\Delta}\left(p^{\prime}\right) \text { and } a_{\Delta}(q), \quad q \in \operatorname{ker} \mu,
$$

are convex subsets of $\Delta$. Now for any element $r$ of $L$ the set $\Delta^{\prime} \cap a_{\Delta}(r)$ coincides with $a_{\Delta^{\prime}}(r)$.

The center of an orthomodular poset $\left(L, \leqslant,,^{\prime}\right)$ is defined by

$$
C(L)=\{p \in L: p C q \text { for all } q \in L\} .
$$

For a probability measure $\mu$ and an element $p$ of the center $C(L)$ of $L$ such that $\mu(p)$ is different from 0 , the element $\mu_{p} \in \mathbb{R}^{L}$ defined by

$$
\mu_{p}(q)=\mu(p \wedge q) / \mu(p), \quad q \in L,
$$

is again a probability measure. A subset $\Delta$ of $\Omega(L)$ is said to be closed under central conditioning provided that $\mu_{p}$ belongs to $\Delta$ for every element $\mu$ of $\Delta$ and every element $p$ of $C(L)$ for which $\mu(p)$ is different from 0 and 1 . We have the following

LEMMA 3.3. Let $\left(L, \leqslant,^{\prime}\right)$ be an orthomodular poset and $\Omega(L)$ the convex set of probability measures on $L$. Every face of $\Omega(L)$ is closed under central conditioning.

Proof. Let $\Delta$ be a face of $\Omega(L)$. Let $\mu$ be an element of $\Delta, p$ an element of $C(L)$ and suppose that $\mu(p)$ is different from 0 and 1 . Now $\mu_{p}$ and $\mu_{p}$, belong to $\Omega(L)$ and for each element $q$ in $L$ we have

$$
\begin{aligned}
\mu(q) & =\mu\left((q \wedge p) \vee\left(q \wedge p^{\prime}\right)\right)=\mu(q \wedge p)+\mu\left(q \wedge p^{\prime}\right) \\
& =\left(\mu(p) \mu_{p}+(1-\mu(p)) \mu_{p^{\prime}}\right)(q) .
\end{aligned}
$$

By the very definition of a face, we conclude that $\mu_{p}$ and $\mu_{p}$, belong to $\Delta$. 
Remark. As a consequence, the sets $\Omega_{\alpha}(L)$ and $\Omega_{c}(L)$ are closed under central conditioning for any orthomodular poset $(L, \leqslant, ')$.

We now turn our attention to the Lebesgue decomposition in the classical context.

Theorem 3.4. Let $(L, \leqslant, ')$ be a Boolean orthomodular poset and let $\Delta$ be a convex subset of $\Omega(L)$.

If $\Delta$ is a subset of $\Omega_{\text {oJP }}(L)$ and if $\Delta$ is closed under central conditioning then $\Delta$ has the positive Lebesgue decomposition property.

Remark. Let $B$ be a field of subsets of a set $X$. Then the triple $\left(B, \subseteq,^{c}\right)$, where $\subseteq$ denotes set-inclusion and $c$ denotes setcomplementation, is a $\sigma$-complete Boolean orthomodular lattice. The classical theorem on the positive Lebesgue decomposition [7] is then recognized in Theorem 3.4. by equating $\Delta$ with $\Omega_{\sigma}(B)$.

Proof of Theorem 3.4. Let $\lambda$ and $\mu$ be elements in $\Delta$ and let $\left(p_{l}\right)_{i=1}^{\infty}$ be a sequence in $\operatorname{ker} \lambda$ such that

$$
\lim _{i \rightarrow \infty} \mu\left(p_{i}\right)=\sup \{\mu(p): p \in \operatorname{ker} \lambda\}
$$

Since $\lambda$ is an element of $\Omega_{\sigma-J P}(L)$ there exists in ker $\lambda$ an upper bound of the set $\left\{p_{i}: i \in \mathbb{N}\right\}$, say $q$. Therefore $\mu$ attains the supremum on $\operatorname{ker} \lambda$ at $q$. We assume that $\mu(q)$ is different from 0 and 1 . Then $\mu_{q}$ and $\mu_{q^{\prime}}$ belong to $\Delta$ and

$$
\mu=\mu(q) \mu_{q}+\mu\left(q^{\prime}\right) \mu_{q^{\prime}}
$$

since $\left(L, \leqslant,^{\prime}\right)$ is Boolean.

Clearly, $\mu_{q}$ is a member of $a_{\Delta}(q)$. If $r$ is any element of $\operatorname{ker} \lambda$, so is $q \vee\left(r \wedge q^{\prime}\right)$. Then

$$
\mu(q) \geqslant \mu\left(q \vee\left(r \wedge q^{\prime}\right)\right)=\mu(q)+\mu\left(r \wedge q^{\prime}\right) \geqslant \mu(q),
$$

thus $\mu\left(r \wedge q^{\prime}\right)=0$. Therefore $\mu_{q^{\prime}}$ is an element of $\bigcap_{p \in k e r \lambda} a_{\Delta}\left(p^{\prime}\right)$. The cases where $\mu(q)$ equals 0 or 1 are easily settled.

Let $(L, \leqslant, ')$ be an orthomodular poset. A subset $\Delta$ of $\Omega(L)$ is said to be unital for $L$ if for each non-zero element $p$ of $L$ there exists an element $\mu$ in $\Delta$ such that

$$
\mu(p)=1 \text {. }
$$

We now proceed to the main result of this paragraph. In an obvious sense it represents a converse to the previous theorem. Applications to operator algebras are considered in the paragraph after next.

THEOREM 3.5. Let $(L, \leqslant, ')$ be an orthocomplete orthomodular poset 
and let $\Delta$ be a unital and convex subset of $\Omega(L)$. If $\Delta$ is a subset of $\Omega_{c-J P}(L)$ and has the positive Lebesgue decomposition property then $\left(L, \leqslant,,^{\prime}\right)$ is Boolean.

Proof. For any element $\lambda$ in $\Delta$ denote with $p_{\lambda}$ the orthocomplement of the largest element in ker $\lambda$. We then have for $r$ an element of $L$

$$
\lambda(r)=1 \Leftrightarrow p_{\lambda} \leqslant r
$$

notice that $p_{\lambda}$ is different from 0 .

Referring to Lemma 3.1., we get

$$
\begin{aligned}
\Delta & =\bigcup_{s \in \text { ker } \lambda} \operatorname{conv}\left(\bigcap_{\text {reker } \lambda} a_{\Delta}\left(r^{\prime}\right) \cup a_{\Delta}(s)\right) \\
& =\operatorname{conv}\left(a_{\Delta}\left(p_{\lambda}\right) \cup a_{\Delta}\left(p_{\lambda}^{\prime}\right)\right)
\end{aligned}
$$

since $a_{\Delta}\left(p_{\lambda}\right)$ coincides with $\bigcap_{r \in k e r \lambda} a_{\Delta}\left(r^{\prime}\right)$.

It follows that for elements $\mu$ and $v$ of $\Delta$

$$
a_{\Delta}\left(p_{\mu}\right)=\operatorname{conv}\left\{\left(a_{\Delta}\left(p_{\mu}\right) \cap a_{\Delta}\left(p_{v}\right)\right) \cup\left(a_{\Delta}\left(p_{\mu}\right) \cap a_{\Delta}\left(p_{v}^{\prime}\right)\right)\right\}
$$

and

$$
a_{\Delta}\left(p_{v}\right)=\operatorname{conv}\left\{\left(a_{\Delta}\left(p_{\mu}\right) \cap a_{\Delta}\left(p_{v}\right)\right) \cup\left(a_{\Delta}\left(p_{\mu}^{\prime}\right) \cap a_{\Delta}\left(p_{v}\right)\right)\right\}
$$

holds true. Let $u$ be a maximal lower bound of $p_{\mu}$ and $p_{v}$, e.g. take the supremum of a maximal orthogonal set of lower bounds of $p_{\mu}$ and $p_{v}$. Similarly, let $v$ and $w$ be maximal lower bounds of $p_{\mu}, p_{\nu}^{\prime}$ and $p_{\mu}^{\prime}, p_{v}$, respectively. Then clearly,

$$
u \perp v \perp w \perp u
$$

and

$$
u \vee v \leqslant p_{\mu}, \quad u \vee w \leqslant p_{v} .
$$

Assume now that $p_{\mu}$ is not equal to $u \vee v$. Then, by orthomodularity, there exists a non-zero element $z$ of $L$ such that

$$
z \perp u \vee v \text { and } z \leqslant p_{\mu} .
$$

Since $\Delta$ is unital there exists an element $K$ in $\Delta$ which evaluates to 1 on $z$ and therefore belongs to $a_{\Delta}\left(p_{\mu}\right)$. If $\kappa$ is neither an element of $a_{\Delta}\left(p_{\mu}\right) \cap$ $a_{\Delta}\left(p_{v}\right)$ nor of $a_{\Delta}\left(p_{\mu}\right) \cap a_{\Delta}\left(p_{v}^{\prime}\right)$ then there exist elements $\xi$ and $\psi$ in $a_{\Delta}\left(p_{\mu}\right) \cap a_{\Delta}\left(p_{v}\right)$ and in $a_{\Delta}\left(p_{\mu}\right) \cap a_{\Delta}\left(p_{v}^{\prime}\right)$, respectively, and a real number $t$ in the open unit interval of the reals such that

$$
\kappa=t \xi+(1-t) \psi
$$

We then have

$$
\xi\left(p_{k}\right)=\psi\left(p_{k}\right)=1
$$


Hence

$$
p_{\xi} \leqslant p_{\kappa} \leqslant z
$$

Now

$$
p_{\xi} \perp u
$$

and also

$$
p_{\xi} \leqslant p_{\mu}, p_{v},
$$

thus

$$
u<u \vee p_{\xi} \leqslant p_{\mu}, p_{v},
$$

a contradiction. The cases where $x$ belongs to $a_{\Delta}\left(p_{\mu}\right) \cap a_{\Delta}\left(p_{v}\right)$ or to $a_{\Delta}\left(p_{\mu}\right) \cap a\left(p_{v}^{\prime}\right)$ are similarly contradictory. This proves that

$$
p_{\mu}=u \vee v .
$$

Corresponding arguments lead to

$$
p_{v}=u \vee w .
$$

To this end we have shown that $p_{\mu} C p_{v}$ for all elements $\mu$ and $v$ in $\Delta$.

Let $p$ be a non-zero element in $L$; then $a_{\Delta}(p)$ is not empty. For any maximal orthogonal subset $D$ of $\left\{p_{\mu}: \mu \in a_{\Delta}(p)\right\}$ the element $p$ is the supremum of $D$; this follows from orthomodularity, orthocompleteness and unitality. Now, for any element $v$ in $\Delta$ we have $p_{v} C D$ and, moreover, $\bigvee_{\in \in D}\left(p_{v} \wedge s\right)$ exists. By a theorem of J. Pool $[16,17]$, it follows that $p$ is compatible with $p_{r}$. A repetition of this argument leads to the conclusion that any pair of elements of $L$ is compatible.

\section{Permanence Properties}

The purpose of this paragraph is to show how the positive Lebesgue decomposition property behaves under the formation of direct sums and direct products of orthomodular posets.

Let $\left(L_{i}, \leqslant_{i},{ }^{\prime}\right)_{i e l}$ be a family of orthomodular posets indexed by the set I. Denote with $1_{i}$ and 0, the greatest and the least element in $\left(L_{i}, \leqslant_{i}\right)$, respectively, for $i$ an element of $I$. Let $\bigcup_{i \in I} L_{i}$ be the set-theoretical sum (disjoint union) of the family $\left(L_{i}\right)_{i \in I}$ and let $j_{i}: L_{i} \rightarrow \bigcup_{i \in I} L_{i}, i \in I$, be the injection maps.

For elements $x$ and $y$ in $\bigcup_{i} L_{i}$ let $p$ and $q$ be elements in $L_{i}$ and $L_{k}$, respectively, such that

$$
x=j_{i}(p) \text { and } y=j_{k}(q) .
$$


Then the relation

$$
\begin{aligned}
x \leqslant y: \Leftrightarrow i=k, p \leqslant i q & \text { or } i \neq k, p=0_{i} \\
& \text { or } i \neq k, q=1_{k}
\end{aligned}
$$

defines a semi-order on $\bigcup_{i} L_{i}$. Let $\sum_{i \in I} L_{i}$ be the quotient of $\bigcup_{i} L_{i}$ with respect to the equivalence relation induced by the semi-order $\leqslant$ and let c: $\bigcup_{i} L_{i} \rightarrow \sum_{i \in I} L_{i}$ be the canonical quotient map. Let $u, v$ be elements of $\sum_{i \in I} L_{i}$ and $x, y$ be elements of $\bigcup_{i} L_{i}$ such that

$$
u=c(x) \text { and } v=c(y) \text {; }
$$

then the relation

$$
u \leqslant v: \Leftrightarrow x \leqslant y
$$

is an order relation on $\sum_{i \in I} L_{i}$. Define for $i \in I$ the mapping $s_{i}: L_{i} \rightarrow \sum_{i \in I} L_{i}$ as the composition of $j_{i}$ and $c$.

Clearly, $\sum_{i \in I} L_{i}$ coincides with the union of the subsets $s_{i}\left(L_{i}\right), i \in I$. For $p$ an element of $L_{i}, q$ an element of $L_{k}$ and the index $i$ different from $k$ we then have

$$
s_{i}(p) \leqslant s_{k}(q) \text { iff } p=0_{i} \text { or } q=1_{k}
$$

Also, if $p$ and $q$ are elements of $L_{l}, i \in I$, then

$$
s_{i}(p) \leqslant s_{i}(q) \text { if and only if } p \leqslant \leqslant_{i} q .
$$

Let $\left(p_{j}\right)$, be a family in $L_{i}$. Then $\bigvee p_{j}$ exists if and only if $\bigvee s_{i}\left(p_{j}\right)$ exists; if either is the case then $s_{i}\left(\bigvee_{j} p_{j}\right)$ coincides with $\bigvee s_{i}\left(p_{j}\right)$. Denote with 1 the element $s_{i}\left(1_{i}\right)$ and with 0 the element $s_{i}\left(0_{i}\right)$, for $i \in I$. Clearly, 1 is the greatest element and 0 is the least element in $\left(\sum_{i \in I} L_{i}, \leqslant\right)$.

Let $u$ be an element in $\sum_{i \in I} L_{i}$ and let $p$ be an element in $L_{i}$ such that $u$ coincides with $s_{i}(p)$; denote with $u^{\prime}$ the element $s_{i}\left(p^{\prime}\right)$. Then the mapping ' $: \sum_{i} L_{i} \rightarrow \sum_{i} L_{i}$ is an orthocomplementation on the poset $\left(\sum_{i} L_{i}, \leqslant\right)$ such that $\left(\sum_{i} L_{i}, \leqslant,^{\prime}\right)$ becomes an orthomodular poset, the direct sum of the family $\left(L_{i}, \leqslant_{i},{ }^{\prime}\right)_{i \in l}$. 
For each $i \in I$ define the mapping $s_{i}^{*}: K\left(\sum_{i} L_{i}\right) \rightarrow \mathbb{R}^{L_{i}}$ by

$$
s_{i}^{*}(\mu)(p)=\mu\left(s_{i}(p)\right)
$$

where $\mu$ is an element of $K\left(\sum_{i} L_{i}\right)$ and $p$ an element of $L_{i}$. One verifies that for each index $i s_{i}^{*}$ maps $K\left(\sum_{i} L_{i}\right)$, resp. $K_{\sigma}\left(\sum_{i} L_{i}\right)$, resp. $K_{c}\left(\sum_{i} L_{i}\right)$, into the cone $K\left(L_{i}\right)$, resp. $K_{\sigma}\left(L_{i}\right)$, resp. $K_{c}\left(L_{i}\right)$, and that it preserves positive combinations of elements of $K\left(\sum_{i} L_{l}\right)$.

Lemma 4.1. Let $\lambda$ and $\mu$ be elements of $K\left(\sum_{i} L_{i}\right)$. Then

(i) $\lambda \perp \mu \Leftrightarrow$ there exists $i \in I$ such that $s_{i}^{*}(\lambda) \perp s_{i}^{*}(\mu)$;

(ii) $\lambda \ll \mu \Leftrightarrow$ for all $i \in I s_{i}^{*}(\lambda) \ll s_{i}^{*}(\mu)$.

Proof. The proof is straightforward.

Let $\mathrm{X}_{i} K\left(L_{i}\right)$ be the set-theoretical product of the family $\left(K\left(L_{i}\right)\right)_{i e I}$ and let $q r_{i}: X_{i} K\left(L_{i}\right) \rightarrow K\left(L_{i}\right), i \in I$, be the projection maps. Define a mapping $\Psi$ from $K\left(\sum_{i} L_{i}\right)$ into $\mathrm{X}_{i} K\left(L_{i}\right)$ by

$$
q r_{i}(\Psi(\mu))=s_{i}^{*}(\mu) \text { for all } i \in I .
$$

Positive $\sigma$-additive and positive completely additive measures are treated accordingly.

LEMMA 4.2. The mapping $\Psi: K\left(\sum_{i} L_{i}\right) \rightarrow \underset{i}{X} K\left(L_{i}\right)$ is injective and its image is equal to the set $\left\{f \in X_{i} K\left(L_{i}\right):\left(q r_{i} f\right)\left(1_{i}\right)=\left(q r_{j} f\right)\left(1_{j}\right)\right.$ for all $i, j \in I\}$. Analogous statements hold for $K_{\sigma}\left(\sum_{i} L_{i}\right)$ and $K_{c}\left(\sum_{i} L_{i}\right)$.

Proof. A verification!

LeMma 4.3. The cone $K\left(\sum_{i} L_{i}\right)$, resp. $K_{o}\left(\sum_{i} L_{i}\right)$, resp. $K_{c}\left(\sum_{i} L_{i}\right)$, consists of the zero measure alone if and only if there exists an index $i$ such that $K\left(L_{i}\right)$, resp. $K_{\sigma}\left(L_{i}\right)$, resp. $K_{c}\left(L_{i}\right)$, consists of the zero measure alone. If either is not the case then for all indices $i$ the mapping $s_{i}^{*}$ maps 
$K\left(\sum_{i} L_{i}\right)$, resp. $K_{o}\left(\sum_{i} L_{i}\right)$, resp. $K_{c}\left(\sum_{i} L_{i}\right)$, onto $K\left(L_{i}\right)$, resp. $K_{o}\left(L_{i}\right)$, resp. $K_{c}\left(L_{i}\right)$.

Proof. (i) Suppose that for all elements $i$ in $I$ the cone $K\left(L_{i}\right)$ is different from $\{0\}$. Select for each index $i$ a non-zero element $\lambda_{\text {, in }} K\left(L_{i}\right)$ and define an element $f$ in $X_{i} K\left(L_{i}\right)$ by

$$
q r_{i}(f)=\lambda_{i} / \lambda_{i}\left(1_{i}\right), \quad i \in I .
$$

Clearly, $f$ is in the image of $\Psi$. If $\Psi^{-1}(f)$ is equal to zero then

$$
\lambda_{i} / \lambda_{l}\left(1_{i}\right)=q r_{i}(f)=s_{i}^{*}(0)=0,
$$

a contradiction.

(ii) Suppose that $K\left(\sum_{i} L_{l}\right)$ has a non-zero element $\mu$. Then $\mu\left(s_{i}\left(1_{i}\right)\right)$ and therefore $s_{i}^{*}(\mu)\left(1_{i}\right)$ is unequal zero for all $i \in I$. This proves that for any index $i s_{i}^{*}(\mu)$ is not the zero measure.

(iii) Let $\lambda_{i}$ be a non-zero element in $K\left(L_{i}\right)$. for each $j \in I, j \neq i$, select a non-zero element $\lambda_{j}$ in $K\left(L_{j}\right)$ and define an element $f$ in $X_{i} K\left(L_{i}\right)$ by

$$
q r_{l}(f)=\lambda_{l}\left(1_{i}\right) \lambda_{l} / \lambda_{l}\left(1_{l}\right), \quad l \in I .
$$

Then $f$ is an element of $\Psi\left(K\left(\sum_{i} L_{i}\right)\right)$ and

$$
s_{i}^{*}\left(\Psi^{-1}(f)\right)=q r_{i}\left(\Psi \Psi^{-1}(f)\right)=\lambda_{i} .
$$

The proofs of the remaining cases are analogous.

THEOREM 4.4. Let $\left(L_{i}, \leqslant_{i},{ }^{\prime}\right)_{l \in l}$ be a family of orthomodular posets and let $\left(\sum_{i} L_{i}, \leqslant,^{\prime}\right)$ be their direct sum. Suppose that $\Omega\left(L_{i}\right)$, resp. $\Omega_{\sigma}\left(L_{i}\right)$, resp. $\Omega_{c}\left(L_{i}\right)$, is not empty for all $i \in I$.

If $\Omega\left(\sum_{i} L_{i}\right)$, resp. $\Omega_{\sigma}\left(\sum_{i} L_{i}\right)$, resp. $\Omega_{c}\left(\sum_{i} L_{i}\right)$, has the positive Lebesgue decomposition property, then so has $\Omega\left(L_{i}\right)$ resp. $\Omega_{\sigma}\left(L_{i}\right)$, resp. $\Omega_{c}\left(L_{i}\right)$, for all $i \in I$.

Proof. Suppose that $\Omega\left(\sum_{i} L_{i}\right)$ has the PLD and is not empty. Let $\lambda, \mu$ be elements of $K\left(L_{i}\right)$; we may assume that $\lambda$ is unequal zero. For each index $j$ different from $i$ select an element $\lambda_{j}$ in $K\left(L_{j}\right)$ such that

$$
\lambda_{j}\left(1_{j}\right)=\lambda\left(1_{i}\right) \text { for all } j \neq i
$$


which is possible by Lemma 4.3. Set

$$
\mu_{j}:=\mu\left(1_{i}\right) \lambda_{j} / \lambda\left(1_{i}\right) \text { for } j \neq i
$$

and define elements $f$ and $g$ in $\underset{i}{X} K\left(L_{l}\right)$ by

$$
\begin{array}{lll}
q r_{i}(f)=\lambda, & q r_{j}(f)=\lambda_{j} \text { for } j \neq i, \\
q r_{i}(g)=\mu, & q r_{j}(g)=\mu_{j} & \text { for } j \neq i .
\end{array}
$$

Obviously, $f$ and $g$ belong to the image of $\Psi$.

Let $\xi$ and $v$ be elements of $K\left(\sum_{i} L_{i}\right)$ such that

$$
\Psi^{-1}(f) \perp \xi, \quad \Psi^{-1}(f) \gg v \quad \text { and } \quad \Psi^{-1}(g)=\xi+v .
$$

By Lemma 4.1., there exists an index $l$ such that

$$
s_{i}^{*}\left(\Psi^{-1}(f)\right) \perp_{l} s_{i}^{*}(\xi) \text {. }
$$

If $l=i$ we conclude that

$$
\begin{aligned}
& \lambda=q r_{i}\left(\Psi \Psi^{-1}(f)\right)=s_{i}^{*}\left(\Psi^{-1}(f)\right) \perp_{i} s_{i}^{*}(\xi), \\
& \lambda=s_{i}^{*}\left(\Psi^{-1}(f)\right) \gg s_{i}^{*}(v), \\
& \mu=q r_{i}\left(\Psi \Psi^{-1}(g)\right)=s_{i}^{*}\left(\Psi^{-1}(g)\right)=s_{i}^{*}(\xi)+s_{i}^{*}(v)
\end{aligned}
$$

and we are done. Suppose now that $l \neq i$ and let $p$ be an element of $L_{l}$ such that

$$
\lambda_{l}(p)=\Psi^{-1}(f)\left(s_{l}(p)\right)=s_{l}^{*}\left(\Psi^{-1}(f)\right)(p)=0=s_{l}^{*}(\xi)\left(p^{\prime}\right) .
$$

Then

$$
\begin{aligned}
0 & =\lambda_{l}(p)=\mu_{l}(p)=\Psi^{-1}(g)\left(s_{l}(p)\right)=\xi\left(s_{l}(p)\right)+v\left(s_{l}(p)\right) \\
& =\xi\left(s_{l}(p)\right) .
\end{aligned}
$$

Therefore $\xi$ is equal to zero and $\Psi^{-1}(f) \gg \Psi^{-1}(g)$. Then clearly

$$
\lambda \perp 0, \lambda \gg \mu \text { and } \mu=\mu+0 .
$$

The proofs for the positive $\sigma$-additive measures as well as the positive completely additive measures are similar.

There are examples of direct sums $\sum_{i} L_{i}$ with infinitely many summands $L_{i}$ different from $\left\{0_{i}, 1_{i}\right\}$ and $\Omega\left(\sum_{i} L_{i}\right)$ having the positive Lebesgue decomposition property. However we have:

TheOREM 4.5. Let $\left(L_{i}, \leqslant_{i},{ }^{\prime}\right)_{i \in I}$ be a family of orthomodular posets indexed by the set $I$. Suppose that $L_{i}$ is different from $\left\{0_{i}, 1_{i}\right\}$ and that 
$\Omega\left(L_{i}\right)$, resp. $\Omega_{\sigma}\left(L_{i}\right)$, resp. $\Omega_{c}\left(L_{i}\right)$, is unital for all $i \in I$. If $\Omega\left(\sum_{i} L_{i}\right)$, resp. $\Omega_{\sigma}\left(\sum_{i} L_{i}\right)$, resp. $\Omega_{c}\left(\sum_{i} L_{i}\right)$, has the positive Lebesgue decomposition property then the indexing set $I$ is finite.

Proof. Assume that $\Omega\left(\sum_{i} L_{i}\right)$ has the PLD and that $I$ is infinite. Let $I^{\prime}$ be a countably infinite subset of $I$ and let $n \in N \rightarrow i(n) \in I^{\prime}$ be a strict enumeration of $l^{\prime}$. For each natural number $n$ select an element $p_{n}$ in $L_{i(n)}-\left\{0_{i(n)}, 1_{i(n)}\right\}$ and elements $\lambda_{n}, \mu_{n}$ in $\Omega\left(L_{i(n)}\right)$ such that

$$
\lambda_{n}\left(p_{n}\right)=1=\mu_{n}\left(p_{n}^{\prime}\right) \text {. }
$$

Define elements $f$ and $g$ in $X K\left(L_{i}\right)$ by

$$
q r_{i(n)}=\lambda_{n} \text { for } n \in \mathbf{N} \text { and } q r_{j}(f)=\lambda_{j} \text { for } j \in I-I^{\prime},
$$

where $\lambda_{j}$ is an arbitrary element of $\Omega\left(L_{j}\right)$;

$$
q r_{i(n)}(g)=(1 / n) \lambda_{n}+(1-1 / n) \mu_{n} \text { for } n \in \mathbf{N}
$$

and

$$
q r_{j}(g)=\lambda_{j} \text { for } j \in I-I^{\prime} .
$$

Clearly, $f$ and $g$ belong to the image of $\Psi$.

Let $\xi$ and $v$ be elements of the cone $K\left(\sum_{i} L_{i}\right)$ such that

$$
\Psi^{-1}(f) \perp v, \quad \Psi^{-1}(f) \gg \xi \quad \text { and } \quad \Psi^{-1}(g)=v+\xi .
$$

For all $n \in \mathbf{N} \Psi^{-1}(f)\left(s_{i(n)}\left(p_{n}^{\prime}\right)\right)$ is equal to zero, hence

$$
\xi\left(s_{i(n)}\left(p_{n}^{\prime}\right)\right)=0, \quad n \in \mathbf{N} \text {. }
$$

Now, for all $n \in \mathbf{N}$,

$$
\begin{aligned}
\xi(1) & =\xi\left(s_{i(n)}\left(p_{n}^{\prime}\right)\right)+\xi\left(s_{i(n)}\left(p_{n}\right)\right)=\xi\left(s_{i(n)}\left(p_{n}\right)\right) \\
& \leqslant \Psi^{-1}(g)\left(s_{i(n)}\left(p_{n}\right)\right)=1 / n,
\end{aligned}
$$

thus $\xi$ is the zero measure and therefore $\Psi^{-1}(f) \perp \Psi^{-1}(g)$. By Lemma 4.1 there exists an index $j$ in $I$ and an element $q$ in $L_{j}$ such that

$$
s_{j}^{*}\left(\Psi^{-1}(f)\right)(q)=0=s_{j}^{*}\left(\Psi^{-1}(g)\right)\left(q^{\prime}\right) .
$$

If $j$ is an element of $I-I^{\prime}$ then

$$
\lambda_{j}(q)=0=\lambda_{j}\left(q^{\prime}\right)
$$

hence $\lambda_{j}\left(1_{j}\right)=0$. If $j=i(n)$ for some $n \in N$ then

$$
\lambda_{n}(q)=0=(1 / n) \lambda_{n}\left(q^{\prime}\right)+(1-1 / n) \mu_{n}\left(q^{\prime}\right),
$$

hence $\lambda_{n}\left(q^{\prime}\right)=0$, thus $\lambda_{n}\left(1_{i(n)}\right)=0$. Therefore in both cases we get a 
contradiction. The balance of the assertion is taken care of in a similar way.

THEOREM 4.6. Let $\left(L_{i}, \leqslant_{i},{ }^{\prime}\right)_{i \in I}$ be a finite family of orthomodular posets.

If $\Omega\left(L_{i}\right)$, resp. $\Omega_{\sigma}\left(L_{i}\right)$, resp. $\Omega_{c}\left(L_{i}\right)$, has the positive Lebesgue decomposition property for all $i \in I$, then so has $\Omega\left(\sum_{i} L_{i}\right)$, resp. $\Omega_{\sigma}\left(\sum_{i} L_{i}\right), \operatorname{resp} . \Omega_{c}\left(\sum_{i} L_{i}\right)$.

Proof. Suppose that $\Omega\left(L_{i}\right)$ has the PLD for all $i \in I$. We may assume that $\Omega\left(\sum_{i} L_{i}\right)$ is not empty. Let $\lambda, \mu$ be elements of $K\left(\sum_{i} L_{i}\right)$. Then for each index $i$ there exist elements $\xi_{i}, v_{i}$ of $K\left(L_{l}\right)$ such that

$$
s_{i}^{*}(\lambda) \perp_{i} \xi_{i}, \quad s_{i}^{*}(\lambda) \gg v_{i} \text { and } s_{i}^{*}(\mu)=\xi_{i}+v_{i} .
$$

Let $k$ be an index such that

$$
v_{k}\left(1_{k}\right) \leqslant v_{i}\left(1_{i}\right) \text { for all } i \in I .
$$

If $v_{k}\left(1_{k}\right)$ is strictly greater than zero we define an element $f$ in $\mathrm{X}_{i} K\left(L_{i}\right)$ through

$$
q r_{i}(f)=v_{k}\left(1_{k}\right) v_{i} / v_{i}\left(1_{l}\right), \quad i \in I .
$$

Then $f$ is in the image of $\Psi$.

For any element $i$ in $I$ and any element $p$ in $L_{i}$ we have

$$
\begin{aligned}
\left(\mu-\Psi^{-1}(f)\right)\left(s_{i}(p)\right) & =s_{i}^{*}(\mu)(p)-v_{k}\left(1_{k}\right) v_{i}(p) / v_{i}\left(1_{i}\right) \\
& \geqslant s_{i}^{*}(\mu)(p)-v_{i}(p)=\xi_{l}(p) \geqslant 0
\end{aligned}
$$

Hence $\mu-\Psi^{-1}(f)$ is an element of $K\left(\sum_{i} L_{i}\right)$. Moreover, $\lambda \perp(\mu-$ $\left.\Psi^{-1}(f)\right)$ since

$$
s_{k}^{*}\left(\mu-\Psi^{-1}(f)\right)=s_{k}^{*}(\mu)-v_{k}\left(1_{k}\right) v_{k} / v_{k}\left(1_{k}\right)=\xi_{k}
$$

and $\xi_{k} \perp s_{k}^{*}(\lambda)$. Also, $\lambda \gg \Psi^{-1}(f)$ since $s_{i}^{*}(\lambda) \gg v_{i}$ implies that

$$
s_{i}^{*}(\lambda) \gg v_{k}\left(1_{k}\right) v_{i} / v_{i}\left(1_{i}\right)=s_{i}^{*}\left(\Psi^{-1}(f)\right) \text {, for all } i \in I \text {. }
$$

If $v_{k}\left(1_{k}\right)$ equals zero, then $v_{k}$ is equal to zero and $\left(s_{k}^{*}(\mu), s_{k}^{*}(\lambda)\right)$ forms a singular pair. Therefore $\mu \perp \lambda$. The proof for the $\sigma$-additive measures as well as the completely additive measures is similar.

We now turn our attention to direct products of orthomodular posets. Let $\left(L_{i}, \leqslant_{i},{ }^{\prime}()_{i e I}\right.$ be a family of orthomodular posets indexed by the set $I$. 
Let $\mathrm{X} L_{i}$ be the set-theoretical product (Cartesian product) of the family $\left(L_{i}\right)_{i}$ and let $p r_{i}: X L_{i} \rightarrow L_{i}, i \in I$, be the projection maps.

Let $u, v$ be elements of $X_{i} L_{i}$; define an order relation in $X_{i} L_{i}$ by

$$
u \leqslant v: \Leftrightarrow p r_{i}(u) \leqslant p r_{i}(v) \text { for all } i \in I .
$$

Then the element 1 , resp. 0 , defined by

$$
p r_{i}(1)=1_{i} \text {, resp. } p r_{i}(0)=0_{i}, \text { for all } i \in I \text {, }
$$

is the greatest, resp. the least, element in the poset $\left(X_{i} L_{i}, \leqslant\right)$. For any element $u$ in $\underset{i}{X} L_{l}$ define an element $u^{\prime}$ in $X_{i} L_{i}$ through

$$
p r_{i}\left(u^{\prime}\right)=\left(p r_{i}(u)\right)^{\prime}, \text { for all } i \in I
$$

Then the mapping ': $\underset{i}{X} L_{i} \rightarrow \underset{i}{X} L_{i}$ is an orthocomplementation which makes the triple $\left(X_{i} L_{i}, \leqslant,^{\prime}\right)$ into an orthomodular poset, the direct product of the family $\left(L_{i}, \leqslant_{i},{ }^{\prime} i\right)_{i \in I}$.

For each index $i$ the mapping $p r_{i}$ is surjective and preserves order as well as orthocomplementation. Let $\left(u_{k}\right)_{k}$ be a family of elements of $\underset{i}{X} L_{i}$. Then $\bigvee u_{k}$ exists if and only if $\bigvee p r_{i}\left(u_{k}\right)$ exists for all $i \in I$; if either is the case then $p r_{i}\left(\bigvee u_{k}\right)$ coincides with $\bigvee_{k} p r_{i}\left(u_{k}\right)$ for all $i \in I$.

Define a mapping $e_{i}: L_{i} \rightarrow X_{i} L_{i}$, for all $i \in I$, through

$$
\operatorname{pr}_{j}\left(e_{i}(p)\right)= \begin{cases}p, & \text { if } i=j, \\ 0_{j}, & \text { if } i \neq j .\end{cases}
$$

Clearly, for each index $i$ the mapping $e_{i}$ is injective and preserves orthogonality. Also, for elements $p$ and $q$ in $L_{l}$

$$
p \leqslant{ }_{l} q \Leftrightarrow e_{i}(p) \leqslant e_{l}(q)
$$

Let $\left(p_{k}\right)_{k}$ be a family of elements in $L_{i}$. Then $\bigvee p_{k}$ exists if and only if $\bigvee e_{i}\left(p_{k}\right)$ exists; if either is the case then $e_{i}\left(Y p_{k}\right)$ coincides with $e_{i}\left(p_{k}\right)$. Let $I^{\prime}$ be a non-empty subset of $I$ and select for each $i \in I$ an element $p_{i}$ of $L_{i}$. Then $\left\{e_{i}\left(p_{i}\right): i \in I^{\prime}\right\}$ is an orthogonal set and the element $u$ 
in $\underset{i}{X} L_{i}$ defined by

$$
p r_{i}(u)= \begin{cases}p_{i}, & i \in I^{\prime} \\ 0_{i}, & i \notin I^{\prime}\end{cases}
$$

is the supremum of this set. Notice that $\left\{e_{i}\left(1_{i}\right): i \in I\right\}$ is a maximal orthogonal set in $X_{i} L_{i}-\{0\}$.

For each $i \in I$ define mappings $p r_{i}^{*}: K\left(L_{i}\right) \rightarrow \mathbb{R}_{i}^{X} L_{i}$ and $e_{i}^{*}: K\left(X_{i} L_{i}\right) \rightarrow \mathbb{R}^{L_{i}}$ by

$$
p r_{i}^{*}(\lambda)(u)=\lambda\left(p r_{i}(u)\right), \quad \lambda \in K\left(L_{i}\right), \quad u \in X_{i} L_{i}
$$

and

$$
e_{i}^{*}(\mu)(p)=\mu\left(e_{i}(p)\right), \quad \mu \in K\left(X_{i} L_{i}\right), \quad p \in L_{i} .
$$

One verifies that the image of $K\left(L_{i}\right)$, resp. $K_{\sigma}\left(L_{i}\right)$, resp. $K_{c}\left(L_{i}\right)$, under the mapping $p r_{i}^{*}$ is contained in $K\left(X_{i} L_{i}\right)$, resp. $K_{\sigma}\left(X_{i} L_{i}\right)$, resp. $K_{c}\left(X_{i} L_{i}\right)$. Similarly, the mapping $e_{i}^{*}$ sends the cone $K\left(X_{i} L_{i}\right)$, resp. $K_{\sigma}\left(X_{i} L_{i}\right)$, resp. $K_{c}\left(X_{i} L_{i}\right)$, into $K\left(L_{i}\right)$, resp. $K_{o}\left(L_{i}\right)$, resp. $K_{c}\left(L_{i}\right)$, for all $i \in I$. Both mappings preserve positive combinations of positive measures and the composition of $p r_{i}^{*}$ with $e_{i}^{*}$ equals the identity mapping on $K\left(L_{i}\right)$ for all $i \in I$.

Lemma 4.7. (i) Let $\lambda, \mu$ be elements in $K\left(\mathrm{X}_{i} L_{i}\right)$. Then $\lambda \ll \mu$ implies $e_{i}^{*}(\lambda) \ll e_{i}^{*}(\mu)$ and $\lambda \perp \mu$ implies $e_{i}^{*}(\lambda) \perp e_{i}^{*}(\mu)$ for all $i \in I$.

(ii) Let $\xi$, $v$ be elements in $K\left(L_{i}\right)$. Then $\xi \ll v$ implies $p r_{i}^{*}(\xi) \ll p r_{i}^{*}(v)$ and $\xi \perp v$ implies $p r_{i}^{*}(\xi) \perp p r_{i}^{*}(v)$ for all $i \in I$.

Proof. (i) Let $\lambda, \mu$ be elements in $K\left(\mathrm{X}_{i} L_{i}\right)$ with $\lambda \perp \mu$. Then there exists an element $u$ in $X_{i} L_{i}$ such that

$$
\lambda(u)=0=\mu\left(u^{\prime}\right) .
$$

Since

$$
\left(e_{i} \circ p r_{i}\right)(u) \leqslant u \text { and }\left(e_{i} \circ p r_{i}\right)\left(u^{\prime}\right) \leqslant u^{\prime}
$$

we conclude that

$$
0=\lambda\left(\left(e_{i}^{\circ} \circ r_{i}\right)(u)\right)=e_{i}^{*}(\lambda)\left(p r_{i}(u)\right)
$$


and similarly

$$
0=e_{i}^{*}(\mu)\left(p r_{i}\left(u^{\prime}\right)\right)=e_{i}^{*}(\mu)\left(p r_{i}(u)^{\prime}\right) .
$$

(ii) Let $\xi$ and $v$ be elements in $K\left(L_{i}\right)$ with $\xi \perp v$; then there exists an element $p$ in $L_{i}$ such that

$$
\xi(p)=0=v\left(p^{\prime}\right) \text {. }
$$

Clearly, $\operatorname{pr}_{i}^{*}(\xi)\left(e_{i}(p)\right)=0$. By orthomodularity, the orthocomplement of $e_{i}(p)$ is equal to $e_{i}\left(p^{\prime}\right) \vee \underset{j \neq i, j \in I}{\bigvee} e_{j}\left(1_{j}\right)$ and we have

$$
\begin{aligned}
\operatorname{pr}_{i}^{*}(v)\left(e_{i}\left(p^{\prime}\right) \vee \bigvee_{j \neq i} e_{j}\left(1_{j}\right)\right)=v\left(\left(p r_{i} \circ e_{i}\right)\left(p^{\prime}\right)\right) & \\
& +v\left(p r_{i}\left(\bigvee_{j \neq i} e_{j}\left(1_{j}\right)\right)\right)=v\left(p^{\prime}\right)+v\left(0_{i}\right)=0 .
\end{aligned}
$$

THEOREM 4.8. Let $\left(L_{i}, \leqslant_{l},{ }^{\prime}\right)_{i \in l}$ be a family of orthomodular posets and let $\left(\mathrm{X}_{i} L_{i}, \leqslant, '\right)$ be their direct product.

If $\Omega\left(X_{i} L_{i}\right)$, resp. $\Omega_{\sigma}\left(X_{i} L_{i}\right)$, resp. $\Omega_{c}\left(X_{i} L_{i}\right)$, has the positive Lebesgue decomposition property, then so has $\Omega\left(L_{i}\right)$, resp. $\Omega_{o}\left(L_{i}\right)$, resp. $\boldsymbol{\Omega}_{c}\left(L_{i}\right)$, for all $i \in I$.

Proof. This is an immediate consequence of Lemma 4.7 and the previous remarks.

Let $(i(n))_{n \in N}$ be a sequence in the indexing set $I$. For each natural number $n$ let $\mu_{n}$ be an element of $K\left(L_{i(n)}\right)$, resp. $K_{\sigma}\left(L_{i(n)}\right)$, resp. $K_{c}\left(L_{i(n)}\right)$, such that

$$
\sum_{n=1}^{m} \mu_{n}\left(1_{i(n)}\right) \leqslant M \text { for all } m \in \mathbf{N}
$$

and some positive scalar $M$. Then the $\tau$-limit of the sequence

$$
\left(\sum_{n=1}^{m} p r_{i(n)}^{*}\left(\mu_{n}\right)\right)_{m \in N}
$$

exists and belongs to $K\left(\underset{i}{X} L_{i}\right)$, resp. $K_{\sigma}\left(\underset{i}{X} L_{i}\right)$, resp. $K_{c}\left({\underset{X}{i}}_{L_{i}}\right),[19$, thm. 2.2.]. Conversely we have

THEOREM 4.9. Let I be a non-empty set, resp. a non-empty countable set. Let $\left(L_{i}, \leqslant_{i},{ }^{\prime}\right)_{i e l}$ be a family of orthomodular posets indexed by the set I. Then for each element $\mu$ in $K_{c}\left(X_{i} L_{i}\right)$, resp. $K_{\sigma}\left(X_{i} L_{i}\right)$, there exists a 
sequence $(i(n))_{n \in N}$ in $I$ and for each natural number $n$ there exists an element $\mu_{n}$ in $K_{c}\left(L_{i(n)}\right)$, resp. $K_{\sigma}\left(L_{i(n)}\right)$, such that

$$
\mu=\tau-\lim _{m \rightarrow \infty} \sum_{n=1}^{m} p r_{i(n)}^{*}\left(\mu_{n}\right) .
$$

If $I$ is a non-empty finite set and $\mu$ an element of $K\left(X_{i} L_{i}\right)$, then for each $i \in I$ there exists an element $\mu_{i}$ in $K\left(L_{i}\right)$ such that

$$
\mu=\sum_{i \in I} p r_{i}^{*}\left(\mu_{i}\right)
$$

Proof. We only consider the case where $I$ is an infinite set and $\mu$ a non-zero element of $K_{\mathrm{c}}\left(\mathrm{X}_{i} L_{i}\right)$. Since $\left\{\boldsymbol{e}_{i}\left(1_{i}\right): i \in I\right\}$ is a maximal orthogonal set in $X_{i} L_{i}-\{0\}$ we conclude that the subset

$$
F:=\left\{i \in I: \mu\left(e_{i}\left(1_{i}\right)\right) \neq 0\right\}
$$

is non-empty and countable. We may assume that $F$ is infinite. Let $n \in \mathbb{N} \rightarrow i(n) \in F$ be a strict enumeration of $F$. Since

$$
\sum_{n=1}^{m} e_{i(n)}^{*}(\mu)\left(1_{i(n)}\right)=\sum_{n=1}^{m} \mu\left(e_{i(n)}\left(1_{i(n)}\right)\right) \leqslant \mu(1)
$$

we conclude that

$$
\tau-\lim _{m \rightarrow \infty} \sum_{n=1}^{m} p r_{i(n)}^{*}\left(e_{i(n)}^{*}(\mu)\right)
$$

exists and belongs to $K_{c}\left(X_{i} L_{i}\right)$.

It then follows for $u$ an element of $X_{i} L_{i}$ that

$$
\begin{aligned}
\lim _{m \rightarrow \infty} \sum_{n=1}^{m} p r_{i(n)}^{*}\left(e_{i(n)}^{*}(\mu)\right)(u)=\lim _{m \rightarrow \infty} \mu\left(\bigvee_{n=1}^{m}\left(e_{i(n)}{ }^{\circ} p r_{i(n)}\right)(u)\right) \\
=\mu\left(\bigvee_{n=1}^{\infty}\left(e_{i(n)}{ }^{\circ} p r_{i(n)}\right)(u)\right)+\mu\left(\bigvee_{i \in F}\left(e_{i} \circ p r_{i}\right)(u)\right) \\
=\mu\left(\bigvee_{i \in I}\left(e_{i}^{\circ} p r_{i}\right)(u)\right)=\mu(u)
\end{aligned}
$$

since $\mu$ is completely additive.

Remark. The representation of a positive $\sigma$-additive measure on the direct product as given in the first part of Theorem 4.9 is also valid if $I$ is a non-real-measurable set [14]. 
THEOREM 4.10. Let I be a non-empty set, resp. a non-empty countable set, resp. a non-empty finite set. Let $\left(L_{i}, \leqslant_{i},{ }^{\prime}\right)_{i \in l}$ be a family of orthomodular posets indexed by the set $I$ and let $\left(X_{i} L_{i}, \leqslant, '\right)$ be their direct product.

If $\Omega_{c}\left(L_{i}\right)$, resp. $\Omega_{o}\left(L_{i}\right)$, resp. $\Omega\left(L_{i}\right)$, has the positive Lebesgue decomposition property for all $i \in I$, then so has $\Omega_{c}\left(X_{i} L_{i}\right)$, resp. $\Omega_{\sigma}\left(X_{i} L_{i}\right), \operatorname{resp.} \Omega\left(X_{i} L_{i}\right)$.

Proof. Suppose that $I$ is an infinite set and that $\Omega_{c}\left(L_{i}\right)$ has the PLD for all $i \in I$. Let $\lambda, \mu$ be elements of $K_{c}\left(X_{i} L_{i}\right)$. Then, by Theorem 4.8 and after the obvious manipulations, there exists a sequence $(i(n))_{n \in N}$ in $I$ and to each $n \in \mathbf{N}$ there exist elements $\lambda_{n}, \mu_{n}$ in $K_{c}\left(L_{i(n)}\right)$ such that

$$
\lambda=\tau-\lim _{m \rightarrow \infty} \sum_{n=1}^{m} p r_{i(n)}^{*}\left(\lambda_{n}\right)
$$

and

$$
\mu=\tau-\lim _{m \rightarrow \infty} \sum_{n=1}^{m} p r_{i(n)}^{*}\left(\mu_{n}\right)
$$

To each natural number $n$ let $v_{n}, \xi_{n}$ be elements in $K_{c}\left(L_{i(n)}\right)$ such that

$$
\lambda_{n} \perp v_{n}, \quad \lambda_{n} \gg \xi_{n} \text { and } \mu_{n}=v_{n}+\xi_{n}
$$

and define $v$ and $\xi$ as the $\tau$-limits of the sequences $\left(\sum_{n=1}^{m} p r_{i(n)}^{*}\left(v_{n}\right)\right)_{m \in N}$ and $\left(\sum_{n=1}^{m} p r_{i(n)}^{*}\left(\xi_{n}\right)\right)_{m \in N}$, respectively. The elements $v$ and $\xi$ belong to the cone $K_{c}\left(X_{i} L_{i}\right)$ and $\mu$ coincides with their sum. If for an element $u$ in $\underset{i}{X} L_{i} \lambda(u)$ equals zero then

$$
0=p r_{i(n)}^{*}\left(\lambda_{n}\right)(u)=\lambda_{n}\left(p r_{i(n)}(u)\right) \text { for all } n \in \mathbb{N} .
$$

Then

$$
0=\xi_{n}\left(p r_{l(n)}(u)\right)=p r_{i(n)}^{*}\left(\xi_{n}\right)(u)
$$

which implies that $\xi(u)$ coincides with zero.

For each natural number $n$ select an element $p_{n}$ in $L_{i(n)}$ such that

Define

$$
\lambda_{n}\left(p_{n}\right)=0=v_{n}\left(p_{n}^{\prime}\right) .
$$

$$
u:=\bigvee_{n \in \mathbb{N}} e_{t(n)}\left(p_{n}\right)
$$


Then, by orthomodularity,

$$
u^{\prime}=\bigvee_{n \in \mathbb{N}} e_{i(n)}\left(p_{n}^{\prime}\right) \vee \bigvee_{j \neq i(n)} e_{j}\left(1_{j}\right)
$$

Since $\lambda$ and $v$ are completely additive measures we conclude that

$$
\lambda(u)=0=v\left(u^{\prime}\right) .
$$

An orthomodular poset is said to be constructible [11] if it can be constructed from a family of Boolean orthomodular lattices by means of a finite number of applications of the direct sum and the direct product operations. Among others we get the following corollary

COROLARY 4.11. Let $\left(L, \leqslant,^{\prime}\right)$ be a finite constructible orthomodular poset. Then $\Omega(L)$ has the positive Lebesgue decomposition property.

Proof. Note that if $\left(L, \leqslant,,^{\prime}\right)$ is a finite Boolean orthomodular poset, then $\Omega(L)$ equals $\Omega_{\text {o-JP }}(L)$ and therefore, by Theorem 3.4, $\Omega(L)$ has the PLD.

\section{Applications to JBW-Algebras}

We study the main results of $\$ 3$ in the context of JBW-algebras.

A real algebra $A$, not necessarily associative, for which

$$
\begin{gathered}
a \circ b=b \circ a, \\
a \circ\left(b \circ a^{2}\right)=(a \circ b) \circ a^{2}
\end{gathered}
$$

holds true and which is also a Banach space with respect to a norm \|\|$: A \rightarrow \mathbb{P}_{+}$satisfying

$$
\begin{aligned}
\|a \circ b\| & \leqslant\|a\| \cdot\|b\|, \\
\left\|a^{2}\right\| & =\|a\|^{2}
\end{aligned}
$$

and

$$
\left\|a^{2}\right\| \leqslant\left\|a^{2}+b^{2}\right\|
$$

is said to be a JB-algebra.

An element $a$ in $A$ is called positive if there exists an element $b$ such that

$$
a=b \circ b \text {. }
$$

The set $A_{+}$consisting of the positive elements in $A$ forms a generating cone in $A$. An idempotent is an element $p$ in $A$ satisfying

$$
p=p \circ p
$$

$U(A)$ denotes the collection of idempotents of $A$. 
A JB-algebra which is the Banach dual space of a, necessarily unique, Banach space is called a JBW-algebra. A JBW-algebra has a unit, denoted by $e$.

Let $A$ be a JBW-algebra with predual Banach space $A_{*}$. For each element $a$ in $A$, the weak*-continuous linear mapping $U_{a}: A \rightarrow A$ is defined by

$$
U_{a} b=\{a b a\}, \quad b \in A,
$$

where, for elements $a, b$ and $c$ in $A$, the Jordan triple product is defined by

$$
\{a b c\}=a \circ(b \circ c)-b \circ(c \circ a)+c \circ(a \circ b) .
$$

With $\leqslant$ we denote the linear order relation in $A$ induced by the cone $A_{+}$. The zero, resp. the unit, in the algebra $A$ is the least, resp. the greatest, element in the poset $(U(A), \leqslant)$. To each element $a$ in $A$ there is a smallest idempotent $r(a)$ with

$$
r(a) \circ a=a
$$

$r(a)$ is said to be the support of $a$. For convenience we denote with $a^{\prime}$ the element $e-a$. Then the mapping ': $U(A) \rightarrow U(A)$ is an orthocomplementation which makes $\left(U(A), \leqslant,,^{\prime}\right)$ into a complete orthomodular lattice. We have, for elements $p$ and $q$ in $U(A)$,

$$
\begin{aligned}
& p \leqslant q \Leftrightarrow U_{p} U_{q}=U_{p}, \\
& p \perp q \Leftrightarrow U_{p} U_{q}=0, \\
& p C q \Leftrightarrow U_{p} U_{q}=U_{q} U_{p} .
\end{aligned}
$$

Moreover, if $p C q$ then

$$
p \wedge q=U_{p} q=p \circ q
$$

and if $p \perp q$ then

$$
p \vee q=p+q
$$

Let $D$ be a non-empty subset of $U(A)$ then

$$
\bigvee D=\text { weak }^{*}-\lim \left(r\left(\sum_{p \in C} p\right)\right)_{C \in D^{u}, C+\infty}
$$

For details the reader is referred to $[1,8,20]$.

In what follows we consider $A_{*}$ to be embedded into the dual Banach space $A^{*}$ of $A$ under the evaluation map, a linear isometry. An element $x$ in $A_{*}$ is called a normal state on $A$ if $x(a)$ is positive for all positive elements $a$ in $A$ and $x(e)$ equals 1 . The convex set $S(A)$ of normal states on $A$ is referred to as the normal state space of $A$. Denote by $\Phi(x)$ the 
restriction to $U(A)$ of the normal state $x$. Then clearly $\Phi(x)$ is a probability measure on the orthomodular poset $\left(U(A), \leqslant,^{\prime}\right)$. Moreover, a normal state $x$ being a weak*-continuous linear functional on $A$, it follows that $\Phi(x)$ is completely additive. Unitalty of the convex subset $\Phi(S(A))$ of $\Omega(U(A))$ follows from the fact that every JBW-algebra has a non-empty normal state space.

Generally, let $a$ be a positive element smaller than $e$. Using spectral theory one shows that the sequence $\left(a_{n}\right)_{n \in N}$ defined by

$$
a_{n}:=\left(\left(a^{\prime}\right)^{n}\right)^{\prime},
$$

is isotone with upper bound $e$ and converges in the weak*-topology to the support $r(a)$ of $a$.

Now, let $x$ be a normal state which vanishes on the idempotents $p$ and $q$. In the sequence above, equate $a$ with $1 / 2 p+1 / 2 q$. By the CauchySchwarz inequality it follows that $x\left(a_{2 n}\right)$ equals zero for all natural numbers $n$. Therefore

$$
0=x(r(1 / 2 p+1 / 2 q))=x(r(p+q))=x(p \vee q) .
$$

Together with Theorem 2.1.(ii) we conclude that $\Phi(S(A))$ is a subset of $\Omega_{c-J P}(U(A))$.

Let $x$ be a normal state and $p$ an idempotent such that $x(p)$ is different from 0 . Then the functional defined by

$$
x_{p}(a):=x\left(U_{p} a\right) / x(p), \quad a \in A,
$$

is a normal state on $A$ since $U_{p}$ is a weak*-continuous positive map on $A$. It follows at once that $\Phi(S(A))$ is closed under central conditioning.

Anticipating the fact that a JBW-algebra is associative if and only if the mappings $U_{p}, p \in U(A)$, commute pairwise we get the following corollary to the Theorems 3.4 and 3.5:

COROLlaRY 5.1. A JBW-algebra is associative if and only if the collection of normal states, considered as probability measures on the orthomodular poset of idempotents, has the positive Lebesgue decomposition property.

\section{REFERENCES}

1. E. M. Alfsen and F. W. Shultz, 'On Non-commutative Spectral Theory and Jordan Algebras', Proc. London Math. Soc. 38, (1979) 497.

2. E. G. Beltrametti and G. Cassinelli, The Logic of Quantum Mechanics, Encyclopedia of Mathematics and its Applications, Volume 15, Addison-Wesley, Reading, 1981.

3. H. R. Fischer and G. T. Rüttimann, 'The Geometry of the State Space', in Mathematical Foundations of Quantum Theory, ed. A. R. Marlow, Academic Press, New York, 1978.

4. D. J. Foulis, 'A Note on Orthomodular Lattices', Portugaliae Math. 21, (1962) 65. 
5. D. J. Foulis and C. H. Randall, 'Operational Statistics I. Basic Concepts.', Journal of Mathematical Physics 13, (1972) 1667.

6. S. P. Gudder, Stochastic Methods in Quantum Mechanics, North Holland, New York, 1979.

7. P. R. Halmos, Measure Theory, Van Nostrand Reinhold, Princeton, 1950.

8. H. Hanche-Olsen and E. Størmer, Jordan Operator Algebras, Pitman, Boston, 1984.

9. S. S. Holland Jr., 'An $m$-Orthocomplete Orthomodular Lattice is $m$-Complete', Proc. Amer. Math. Soc. 24, (1970) 716.

10. S. S. Holland Jr., 'A Radon-Nikodym Theorem in Dimension Lattices', Trans. Amer. Math. Soc. 108, (1963) 66.

11. M. F. Janowitz, 'Constructible Lattices', Journal of the Australian Mathematical Sociery 14, (1972) 311.

12. J. Jauch, Foundations of Quantum Mechanics, Addison-Wesley, Reading, 1968.

13. G. Kalmbach, Orthomodular Lattices, Academic Press, London, 1983.

14. V. Manasová and P. Pták, 'On States on the Product of Logies', International Joumal of Theoretical Physics 20, (1981) 451.

15. C. Piron, Foundations of Quantum Physics, Benjamin Inc., Reading, 1976.

16. J. C. T. Pool, Simultaneous Observability and the Logic of Quantum Mechanics, $\mathrm{Ph} . \mathrm{D}$. Thesis, University of Iowa, 1963.

17. G. T. Rüttimann, 'Non-commutative Measure Theory', Habilitationsschrift, Universität Bern, 1980.

18. G. T. Rüttimann, 'Jauch-Piron States', Journal of Mathematical Physics 18, (1977) 189.

19. G. T. Rüttimann, 'Facial Sets of Probability Measures', Probability and Mathematical Statistics, 6, (1985) 99.

20. F. W. Shultz, 'On Normed Jordan Algebras Which Are Banach Dual Spaces', J. Funct. Anal. 31, (1979) 360.

21. N. Zierler, 'Axioms for Non-relativistic Quantum Mechanics', Pacific J. Math. 11, (1961) 1151.

University of Berne,

Institute of Mathematical Statistics,

Sidlerstrasse 5, CH-3012 Bern,

Switzerland 